\title{
Thermal treatment of Rapeseed oil
}

\author{
Shanmugam Palanisamy, Börje S. Gevert \\ Chalmers University of Technology, Göteborg, Sweden \\ Corresponding author.Tel: +4631 7722812,E-mail: shapal@chalmers.se
}

\begin{abstract}
The thermal decomposition of rapeseed oil lowered cetane value of the product through decarboxylation and decarbonylation. In this study the thermal decomposition in rapeseed oil was estimated with different temperatures $\left(300\right.$ to $410^{\circ} \mathrm{C}$ ) with or without hydrogen at 1 bar partial pressure. Initially, the reactor is loaded with glass pellets and then the rapeseed oil was fed into the reactor. At hydrothermal condition of 300 to $410^{\circ} \mathrm{C}$, the formation of oxygenate groups (i.e. esters, acids and aldehydes) were 15 to $30 \%$, while the rest contained thermally cracked hydrocarbons with excluded un-reacted feed. In residue oil, cyclic group formation was observed. The formation of acidic and aldehyde resulted in carbon dioxide and carbon monoxide in outlet gases. The hydroprocess of higher temperatures leaded higher cracking and cyclic groups with more dense and viscous residue oil.
\end{abstract}

Keywords: Hydrodeoxygenation, Decarboxylation, Thermal conversion, Vegetable oil, Bio-fuels.

\section{Introduction}

Recently, traditional oil refineries have started to hydroprocessing vegetable oil and fatty acids. Traditional renewable fuels were (1) fuel production based on a super critical process, (2) bio-ethanol technology and (3) production of FAME. All three fuel sources have received considerable attention over the past decade in order to achieve bio-refinery status. Apart from this research development, hydroprocessing was a friendly and suitable process for existing oil refinery concepts to include biomass as a co-feed. One big advantage is the factor of scale in oil refinery. The cost for processing is low per unit and the biomass can ride on this low cost of production and distribution of the products [1, 2]. During hydroprocessing, water is removed from the carboxylic group from tri-glycerol to give $\mathrm{C}_{18}$ hydrocarbon and propane, is known hydro-deoxygenation. The product had a high cetane value, low density and poor cold flow properties [3].

Thermal decomposition is unfavorable at deoxygenation mechanism. Usually, excess hydrogen partial pressure is needed for deoxygenation because of certain diffusion limitations over films. These limitations favored for thermal effect on carboxylic group to form $\mathrm{CO}_{2}$ and CO. Thermal decomposition of the carboxylic group reduced to $\mathrm{C}_{17}$ and expelled $\mathrm{CO}$ and $\mathrm{CO}_{2}$ in the gas phase, is known as decarboxylation/decarbonylation. Indeed, loss of one carbon at each hydrocarbon chain in decarboxylation resulted in a difference in the cetane value. Also, methylation and water-gas shift reaction (WGS) were evident as part of the catalytic thermal conversion [2- 4]. Eventually, the temperature around 300 to $360^{\circ} \mathrm{C}$ proved feasible for deoxygenation, and some researcher indicate it increases the decomposition $[5,6]$.

Much research on hydroprocessing could explain deoxygenation, thermal conversion, hydration and WGS reaction, but had trouble predicting the exact evaluation of the reaction path due to change in properties of vegetable oil during pre-heating [5-8]. Pre-heating in reactor is common technique for catalytic process. As a part on thermal effect of preheating, we focused only on the thermal effect to identify the modification in vegetable oil with hydrogen as co-feeding at ambient pressure and reinstate the result for future work on our catalytic process. During hydro deoxygenation, the vegetable oil has been subjected with hydrogen into reactor on concurrent flow. This assignment was to characterize the feed before introduce into Hydro deoxygenation catalytic process. 


\section{Experiment}

The experimental setup consisted of a continuous reactor, a feed and product tank, a gas flow meter, pump, a gas chromatography and controllers. The major reaction conditions parameters were liquid hourly space velocity (LHSV $=\mathrm{ml}$ of liquid/g.cat*hr), gas hourly space velocity $\left(\mathrm{GHSV}=\mathrm{ml}\right.$ of gas/g.cat $\left.{ }^{*} \mathrm{hr}\right)$, reaction pressure (bar) and reaction temperature $\left({ }^{\circ} \mathrm{C}\right)$. However, in our experiment, overall reaction conditions were adjusted with constant feed flow on the continuous reactor on a weight basis. The sample and gas outlet were collected under ambient atmospheric conditions. The reaction condition was stabilized through proportional-integralderivative (PID) controllers. The samples were withdrawn subject to a manual time control and all samples were taken under a stabilized system.

\subsection{Reactor}

The experimental setup consisted of a feed tank, fixed-bed reactor, product tank, gas collector and dossier pump. The fixed-bed reactor was fixed with an electric furnace, connected with an instrumental controller to regulate thermal and pressure conditions. The vertical height of the reactor was $619.4 \mathrm{~mm}$, excluding an external clump with bolt; the reactor was filled with 2 mm diameter glass pellets. The maximum temperature, taken at the middle of the reactor, was the indicated temperature point. There are another two more thermocouple connected at inlet and outlet on reactor to monitor the reaction temperature.

\subsection{Analytical work}

The gas outlet and liquid products from the reactor were analyzed with different gas chromatography equipment. The liquid analysis was performed according to ASTM D2887 using a gas chromatograph (GC) (Varian 3400) equipped with a packed column (OV101) and flame ionization detector (FID) with nitrogen as carrier gas. The outlet gas from the reactor was analyzed by the Clarus 500 GC online, which was connected with 600 link switch controllers; the signal was integrated to receive analytical data. Gas analysis consisted of the use of a thermal conductivity detector (TCD) to analyze $\mathrm{CO}, \mathrm{CO}_{2}, \mathrm{CH}_{4}$ and $\mathrm{H}_{2}$, as well as FID for hydrocarbons.

Elemental analysis of C, H and O was analyzed by Karlshamn Kraft AB. The ASTM D 5291 standard test method for instrumental determination of carbon, hydrogen and nitrogen in petroleum products and lubricants was used in our samples. Further, traces of nitrogen, sulphur and other metals in the sample were neglected and oxygen content was estimated from carbon and hydrogen content. The accuracy of the elemental analysis was $\pm 0.4 \mathrm{wt} \%$.

The samples were analyzed with the Perkin-Elmer Spectrum One Fourier Transform Infrared Spectroscopy (FTIR) to identify the internal change in processed vegetable oil. The liquid samples were placed between two plates in pure sodium chloride salt without any bubbles. The mid-infrared was used between wavelengths 400 and $4000 \mathrm{~cm}^{-1}$ to study the fundamental structure of the sample.

\section{Results}

The continuous reactor was heated to $350^{\circ} \mathrm{C}$ temperature, after which vegetable oil was fed into the system. Initially, the feed was supplied for $48 \mathrm{~h}$ without any interruption to make sure the system was clean and to achieve a steady state. After this phase, the reactor conditions were changed systematically to obtain the samples for the appropriate conditions. The 
samples were collected on a weight basis at ambient pressure from a fixed feed rate of 200 $\mathrm{ml} / \mathrm{min}$ over $19 \mathrm{~h}$ for each condition.

The rapeseed oil contains $7 \%$ stearic (18:0), 61.1\% oleic acid (18:1), 20.9\% linoleic acid (18:2) and the rest consisted of omega-3. At $300^{\circ} \mathrm{C}, 5.12 \mathrm{wt} \%$ of free $\mathrm{C}_{17}-\mathrm{COOH}$ and $1 \mathrm{wt} \%$ of other hydrocarbons appeared in the products. In addition to $\mathrm{C}_{17}$, there were traces of lighter hydrocarbon with less than $2 \mathrm{~mol} \%$ and $10 \mathrm{~mol} \%$ of CO in the gas phase (Figure 1). At $330^{\circ} \mathrm{C}$, the carboxylic group increased to $8 \mathrm{wt} \%$ and $2 \mathrm{wt} \%$ of other products. Over $330^{\circ} \mathrm{C}$, there was a steady increase of both - (COO) - and - (CO) - groups can be seen in Table 1, as well as some lighter hydrocarbons. The results of the thermal effect on $(\mathrm{C}=\mathrm{O})-\mathrm{O}-\mathrm{C}$ bond detachment had observed uniform distribution in gas chromatography.

Table 1. Rapeseed oil hydro-treatment at $L H S V=20 \mathrm{mln} / \mathrm{min}, 1$ bar and $H_{2} / o i l=10$. $R$ $(C=O) O$ refers to $B P$ from 340 to $360^{\circ} \mathrm{C}$ while $R-(C=O)$ refers to $B P$ about $330^{\circ} \mathrm{C}$.

\begin{tabular}{ccc}
\hline Temp. $\left({ }^{\circ} \mathbf{C}\right)$ & R-(C=O)O $\mathbf{( w t} \%)$ & R-(C=O) $(\mathbf{w t} \%)$ \\
\hline $\mathbf{3 0 0}$ & 5.12 & 0 \\
$\mathbf{3 3 0}$ & 7.95 & 1.9 \\
$\mathbf{3 5 0}$ & 6.55 & 2.65 \\
$\mathbf{3 7 0}$ & 8.43 & 3.99 \\
$\mathbf{3 9 0}$ & 11,56 & 3,64 \\
\hline
\end{tabular}

High concentration of methane and ethane/ethane in the gas outlet were observed for lower temperatures. The dispersed gas in the liquid sample was not recoverable, so it was difficult to make mass balance for in and out of gas flow. Thus, we avoided dealing with the mass balance and instead just showed the concentration in the gas phases. The changes in flow of liquid in and liquid out were almost the same (variation of 0.01 to $0.05 \mathrm{wt} \%$ ).

\section{Discussion}

In our study the carboxylic groups were mostly converted to free fatty acid and some traces of alcohol and aldehyde between $330^{\circ} \mathrm{C}$ and $350^{\circ} \mathrm{C}$. This finding implies that the carboxyl groups were influenced by temperature and induced both decarbonylation and decarboxylation. However, there was less evidence of glycerol or glycol groups under these conditions. The gas phase contained $\mathrm{CO}$ as the main substitute (Figure 2). The CO outlet shows that the bonding of the carboxyl group was weaker than the $\mathrm{C}-\mathrm{C}$ bond.

The carboxyl groups tended to detach above $350^{\circ} \mathrm{C}$ in high yield as acids, aldehyde, esters and paraffin. The concentration of carboxylic acid was doubled and paraffin concentration increased one- to three-fold for every $20^{\circ} \mathrm{C}$ rise in temperature. The product was distilled in simple distillation at a cut-off of $235^{\circ} \mathrm{C}$, which removes water and lighter naphtha named distillate. The distillate had greenish appearance, with gasoline density and viscosity [9]. It had acidic compound. In figure 2 shows the FTIR result of distillate without water in it. Here, $\mathrm{C}=\mathrm{O}$ groups identified in FTIR mainly of acids while the bottom product consisted of aldehyde and esters. The information in the figure proves that distillate had lighter hydrocarbon with acid group; these conclude that distillate mostly formed from C-C cracking. However, the esters and aldehyde groups had $\mathrm{C}-\mathrm{O}-\mathrm{C}$ - bond breaking, as was evident from the thermal impact. 

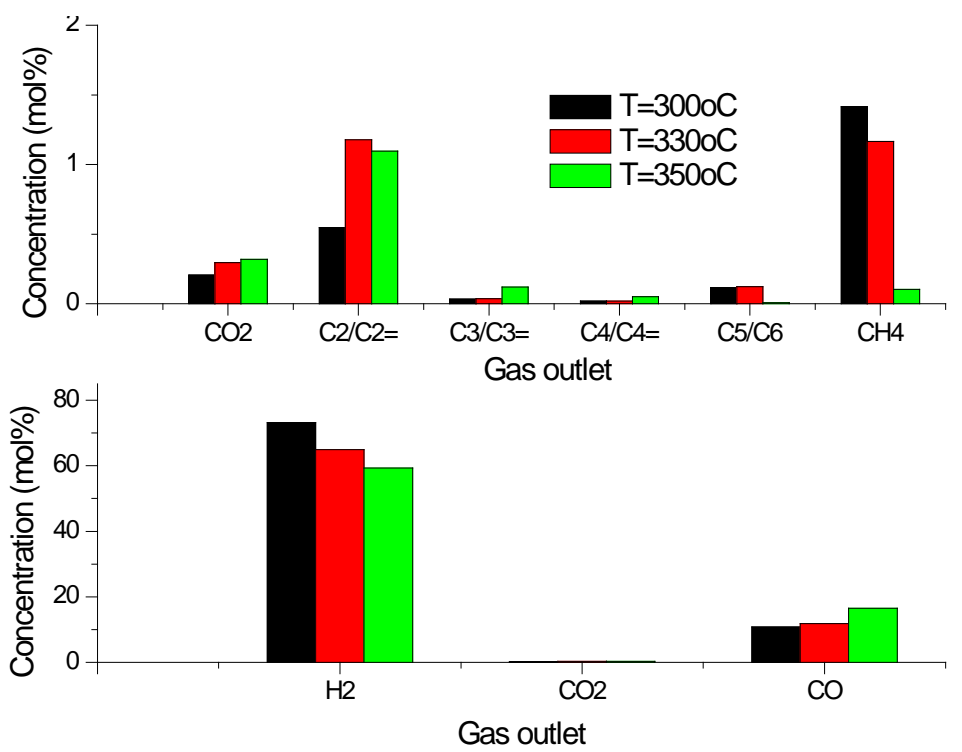

Figure. 1. Rapeseed oil hydro-treatment: gas outlet at $L H S V=20 \mathrm{mln} / \mathrm{min}, 1$ bar and $\mathrm{H}_{2} / \mathrm{oil}$ $=10$.

At higher temperature (above $350^{\circ} \mathrm{C}$ ) the oxygen content reduced between 10 and $12 \%$ into water, $\mathrm{CO}$ and $\mathrm{CO}_{2}$. The distilled sample contained water from 0.04 to $0.1 \mathrm{wt} \%$. The presences of water indicate that the hydroprocess was part in thermal reaction [10].

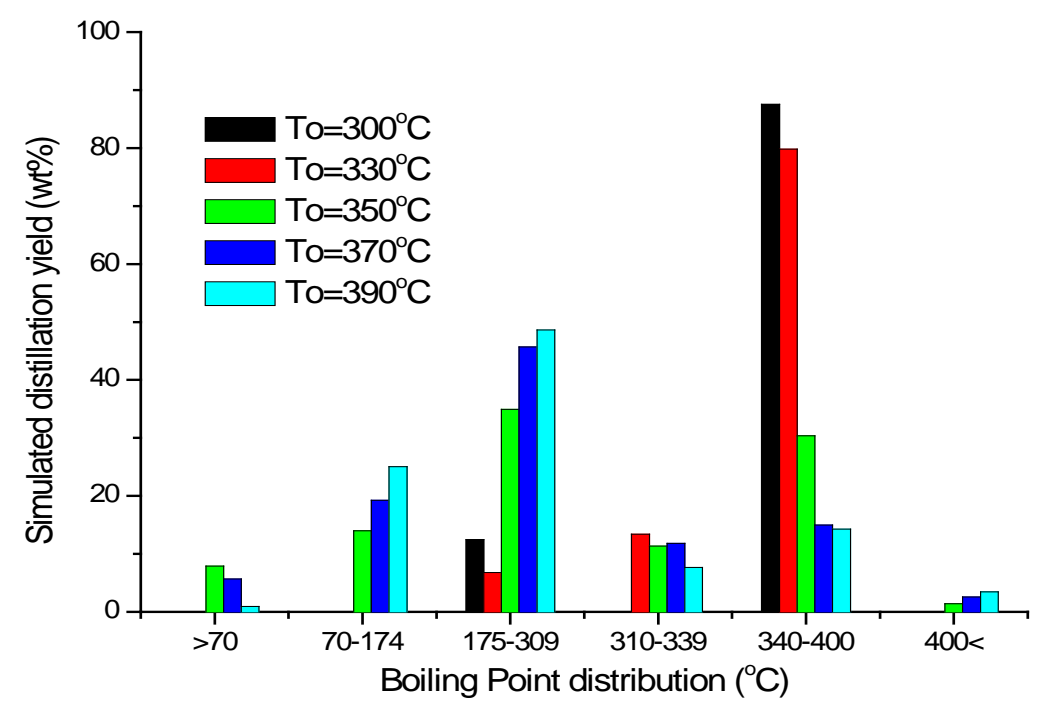

Figure 2. Rapeseed oil hydro-treatment at LHSV $=20 \mathrm{mln} / \mathrm{min}, 1$ bar and $\mathrm{H}_{2} / \mathrm{oil}=10$ excluding untreated rapeseed oil. 


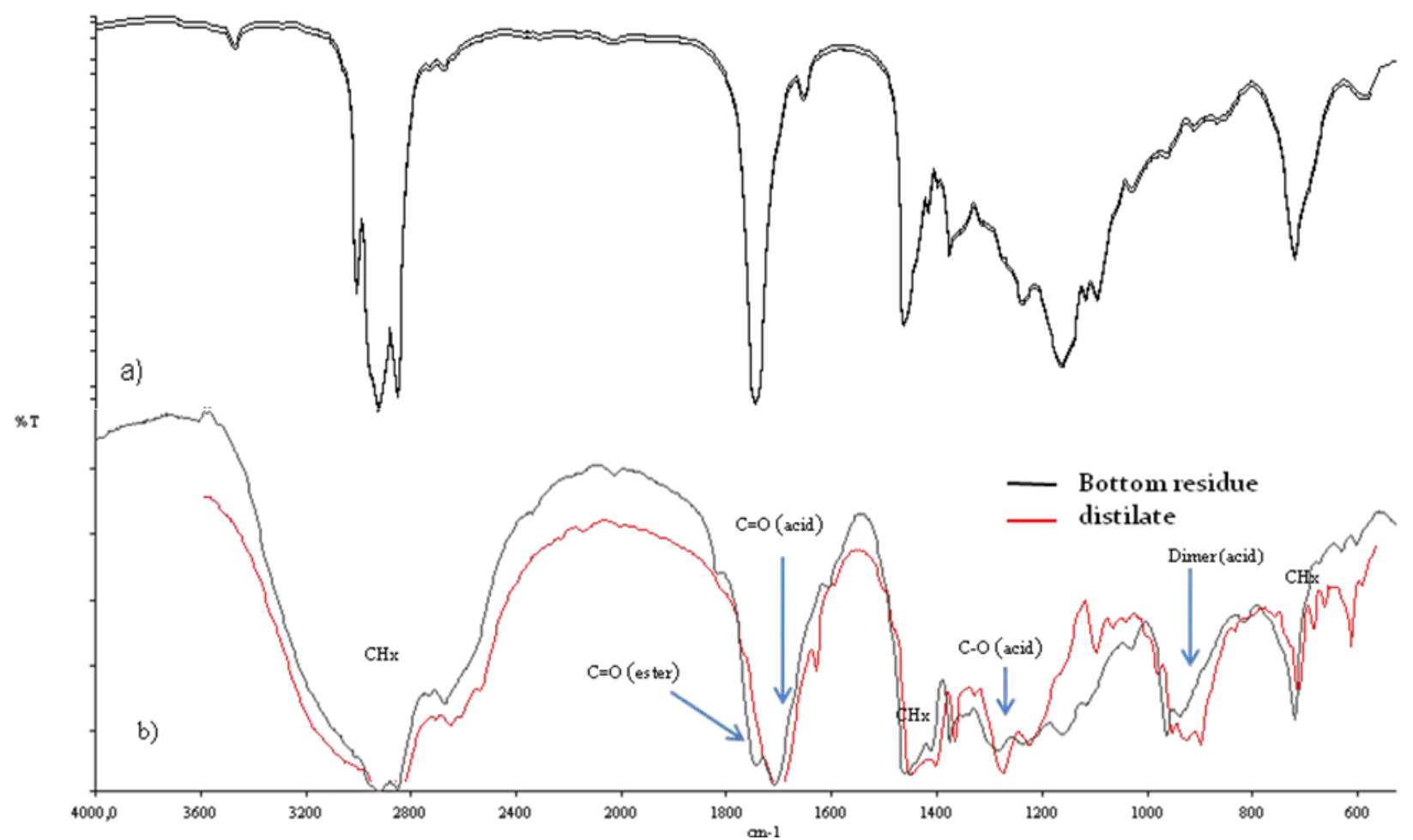

Figure 3. FTIR analysis for distilled product (LHSV $=20 \mathrm{mln} / \mathrm{min}, 1 \mathrm{bar}, \mathrm{H}_{2} / \mathrm{oil}=10 \& \mathrm{~T}=$ $350^{\circ} \mathrm{C}$ ). (a) Rapeseed oil and (b) Simple distillation at a cut-off point of $235^{\circ} \mathrm{C}$.

\section{Conclusion}

The hydroxyl groups in triglycerides were found to be sensitive to temperature. The product variant in different temperatures was dependent on only $\mathrm{CO}$ and water outlet. Of the major gas outlets, $\mathrm{CO}$ dominated the composition at higher temperatures. Thermal cracking of $\mathrm{C}=\mathrm{C}$ bonds between 300 and $350^{\circ} \mathrm{C}$, was minimal in this study. The condition between 330 to $360^{\circ} \mathrm{C}$ indicates that $\mathrm{CO}$ formation has more dominant during thermal decomposition.

\section{References}

[1] S.N. Naik, Vaibhav V. Goud, Prasant K. Rout and Ajay K. Dalai, Production of first and second generation bio-fuels: A comprehensive review, Renewable and Sustainable Energy Reviews 14, Issue 2, February 2010, Pages 578-597.

[2] Ayhan Demirbas, Progress and recent trends in bio-fuels, Progress in Energy and Combustion Science 33 (2007) 1-18.

[3] David Kubic.ka, Lude.k Kaluz', Deoxygenation of vegetable oils over sulfided Ni, Mo and NiMo catalysts, Applied Catalysis A: General 372 (2010) 199-208.

[4] Jeong-Geol Na, Bo Eun Yi, Ju Nam Kim, Kwang Bok Yi, Sung-Youl Park, Jong-Ho Park, Jong-Nam Kim, Chang Hyun Ko, Hydrocarbon production from decarboxylation of fatty acid without hydrogen, Catalysis Today 156 (2010) 44-48.

[5] Bjorn Donnis, Rasmus Gottschalck Egeberg, Peder Blom and Kim Gron Knudsen, Hydro-processing of Bio-oils and Oxygenates to Hydrocarbons. Understanding the Reaction Routes, Top Catalyst 52, 2009, pp. 229 - 240.

[6] Thiam Leng Chew and Subhash Bhatia, Catalytic processes towards the production of bio-fuels in a palm oil and oil palm biomass-based bio-refinery, Bio-resource Technology 99, Issue 17, November 2008, Pages 7911-7922. 
[7] Serdar Yaman, Pyrolysis of biomass to produce fuels and chemical feedstock's, Energy Conversion and Management 45, Issue 5, March 2004, Pages 651-671.

[8] Pavel Šimác.ek, David Kubic.k, Gustav Šebor, Milan Pospíšil, Hydroprocessed rapeseed oil as a source of hydrocarbon-based biodiesel, Fuel 88 (2009) 456-460.

[9] Pavel Šimác.eka, David Kubic.kab, Gustav Šebor, Milan Pospíšil, Fuel properties of hydroprocessed rapeseed oil, Fuel 89 (2010) 611-615.

[10] Marton Krar, Sandor Kovacs, Denes Kallo, Jenö Hancsok, Fuel purpose hydro treating of Sunflower oil on Co/ $\mathrm{Al}_{2} \mathrm{O}_{3}$ catalyst, Bioresource Technology 101 (2010), 9287-9293. 